\title{
Painting Therapy in Cancer Care: A Descriptive Systematic Review
}

\author{
Zhenting Ding, Yanqiong Ouyang, Na Zhang \\ School of Health Sciences, Wuhan University, Wuhan, China \\ Email: dingzhenting@foxmail.com
}

How to cite this paper: Ding, Z.T., Ouyang, Y.Q. and Zhang, N. (2019) Painting Therapy in Cancer Care: A Descriptive Systematic Review. Open Journal of Nursing, 9, 89-102.

https://doi.org/10.4236/ojn.2019.92009

Received: January 10, 2019

Accepted: February 11, 2019

Published: February 14, 2019

Copyright $\odot 2019$ by author(s) and Scientific Research Publishing Inc. This work is licensed under the Creative Commons Attribution International License (CC BY 4.0).

http://creativecommons.org/licenses/by/4.0/ Open Access

\begin{abstract}
Background: Despite art therapy has been widely used in cancer population to improve their physical and mental health, painting therapy, as one form of art therapy, is seldom applied to adult cancer patients independently. Therefore, how painting therapy affects adult cancer patients' health outcomes needed to be explored sufficiently. Objectives: The purpose of this review was to assess and synthesize the latest evidence of painting therapy concerning adult cancer patients, and to provide some inspiration for clinical staff. Methods: A literature research of PubMed, Web of Science, Cochrane Library, EMBASE, CINAHL, PsychoInfo, Psydex, CENTRAL, Google Scholar, British Library, CNKI, Wanfang, CQVIP electronic databases were undertaken with the following key words: art therapy, painting, drawing, cancer, neoplasm, tumor, carcinoma and oncology. A narrative synthesis was used according to PRISMA and the quality of study was assessed using acritical assessment tool. Results: Eleven papers reporting nine different research projects met the inclusion criteria. Generally speaking, painting therapy positively affected health outcomes. The impacts on patients include improvements in quality of life, coping with disease, mental health and somatic symptoms. However, inconsistency still exists. Three studies failed to show positive effects of painting therapy on post-traumatic growth, distress and coping behavior in participants. Conclusion: Robust evidence was found in rehabilitation of patients with various types of cancer. Painting therapy evidently affected cancer patients in a positive direction. However, the application of painting therapy to cancer patients needs to be explored further due to few studies on this topic.
\end{abstract}

\section{Keywords}

Painting Therapy, Cancer Care, Systematic Review 


\section{Introduction}

It is an ongoing challenge to find novel ways to increase cancer awareness and provide support to cancer population. Up to now, complementary and alternative medicine (CAM) in relation to cancer treatment has been widely used. As one of various kinds of CAM, art therapy intended to improve the quality of life of patients. Art materials are used in art therapy for empowering individuals through the self-expression, self-identification and personal conflicts resolution in the presence of a trained art therapist [1] [2]. Art therapy represents a specific type of psycho-social intervention [3], which includes a wide range of approaches and therapeutic applications [4]. Art disciplines, such as music, painting, drawing, collage, sculpture, dance, creative drama or writing, were included in art therapy [5].

Painting therapy is a form of art therapy mediated by painting activities. In 1986, Judy et al. developed a program "We can weekend" to help families to cope with cancer in a form of drawing and painting [6]. From then on, more similar therapies for cancer patients have been developed. Notably, although various positive effects of art therapy with painting therapy and other forms of therapy embedded have been documented, it is difficult to figure out whether painting therapy result in the effect independently. Additionally, painting therapy was mainly developed in adult psychiatric patients and pediatric patients rather than adult cancer population. As a result, there are limited data on painting therapy independently used in cancer patients, and related systematic evaluations on this topic are rare. To our best knowledge, only one overview of art therapy based on painting or drawing was found and painting therapy was just mixed with other forms of art therapy in the included studies [7]. As painting therapy in psycho-oncology is gaining increasing importance, it is necessary to review on this topic.

By summarizing the direction of evidence (positive, uncertain, negative), this review aimed to evaluate whether painting therapy could contribute meaningful impacts on cancer patients, and to explore how painting therapy work. The specific aims of this review include:

1) To synthesize the effect of painting art therapy on health outcomes of adult patients with cancer;

2) To explore the mechanism of painting therapy;

3) To identify the limitations of implemented painting therapy.

\section{Methods}

\subsection{Search Strategy}

The review procedure was guided by the Preferred Reporting Items for Systematic Reviews and Meta-Analyses (PRISMA) statement [8]. The following databases were searched: PubMed, Web of Science, Cochrane Library, EMBASE, CINAHL, PsychoInfo, Psyndex, CENTRAL, Google Scholar, British Library. Other electronic databases such as CNKI, Wanfang database, CQVIP were also 
included. All the databases were retrieved from inception to September 2018 by two researchers independently. The references of included studies were also screened. Note express software was used to organize citations.

Search terms used were: ("tumor" OR "neoplasm" OR "cancer" OR "carcinoma" OR "oncology") for cancer and ("drawing” OR "painting” OR "art”) for painting therapy. Search terms were a combination of MeSH terms and free-text terms. Relevant studies in English or Chinese were reviewed.

\subsection{Selection Criteria}

Inclusion criteria were:

1) Empirical studies with any type of design;

2) Outcomes including quality of life, coping with disease, mental health and somatic symptoms measured;

3) Cancer population aged 18 years or older;

4) Implementing painting therapy intervention;

5) Published in English or Chinese.

Exclusion criteria were:

1) Besides painting therapy, other forms of art therapy involved in the intervention;

2) Lack of full-text literature.

\subsection{Quality Appraisal}

For included studies, the heterogeneity made it inappropriate to use Cochrane Collaboration approach. In order to assess the quality of a diverse group of empirical studies, Hawker developed an appraisal tool to evaluate studies using both qualitative methods and quantitative methods [9]. There are 9 evaluating items in this tool. Each item includes 4 grades: good, fair, poor, very poor (10indicating very poor; 40 indicating good). A total score based on 9items is 360 . The higher the score is, the better the quality of research would be.

\subsection{Data Extraction}

All retrieved articles were double-blind assessed to evaluate suitability for inclusion by two reviewers. Uncertainty was resolved by discussion with the third researcher. Data from the selected articles was extracted: Authors, year of publication, country, participants, study design, age range, intervention outcome measures and methodological issues.

\subsection{Data Analysis}

As Eysenck stated, "Meta-analysis is only properly applicable if the data summarized are homogeneous - that is, treatment, patients, and end points must be similar or at least comparable" [10]. The heterogeneity included various types of design, follow-up duration and different outcomes, which indicated unfeasible to do a statistical meta-analysis. Therefore, data was analyzed using narrative 
synthesis which relied primarily on the use of words and text to summarize and explain the findings of this synthesis [11].

\section{Results}

\subsection{Research Outcomes}

A total of 2077 titles and abstracts were screened, and 319 duplicates were identified. Of the 1758, 1642 were excluded via reviewing titles and abstracts. After reading the remaining 116 full-text articles, 106 articles were excluded because intervention in these studies did not meet the inclusion criteria and articles were not written in English or Chinese. One more study was identified by searching the reference lists. Finally, 11 papers were included [12]-[22]. Three papers reporting different aspects of a project were all included owing to few research papers in this topic up to now [17] [20] [21]. The flow diagram of study selection was shown in Figure 1.

\subsection{Study Characteristics}

Study characteristics were summarized in Table 1. Eleven studies derived from 6 countries: Germany, Turkey, China, Sweden, Israel and Denmark. The current systematic review includes 4 randomized controlled trials, 6 non-randomized controlled trials and 1 qualitative study. Data from the qualitative study was analyzed from 15 drawings and post-drawing interviews with participants using visual and hermeneutic phenomenology [12]. Except for Hammer's study [12], data from other studies were collected through self-report questionnaire. No specific theory was applied to guide intervention in these included studies.

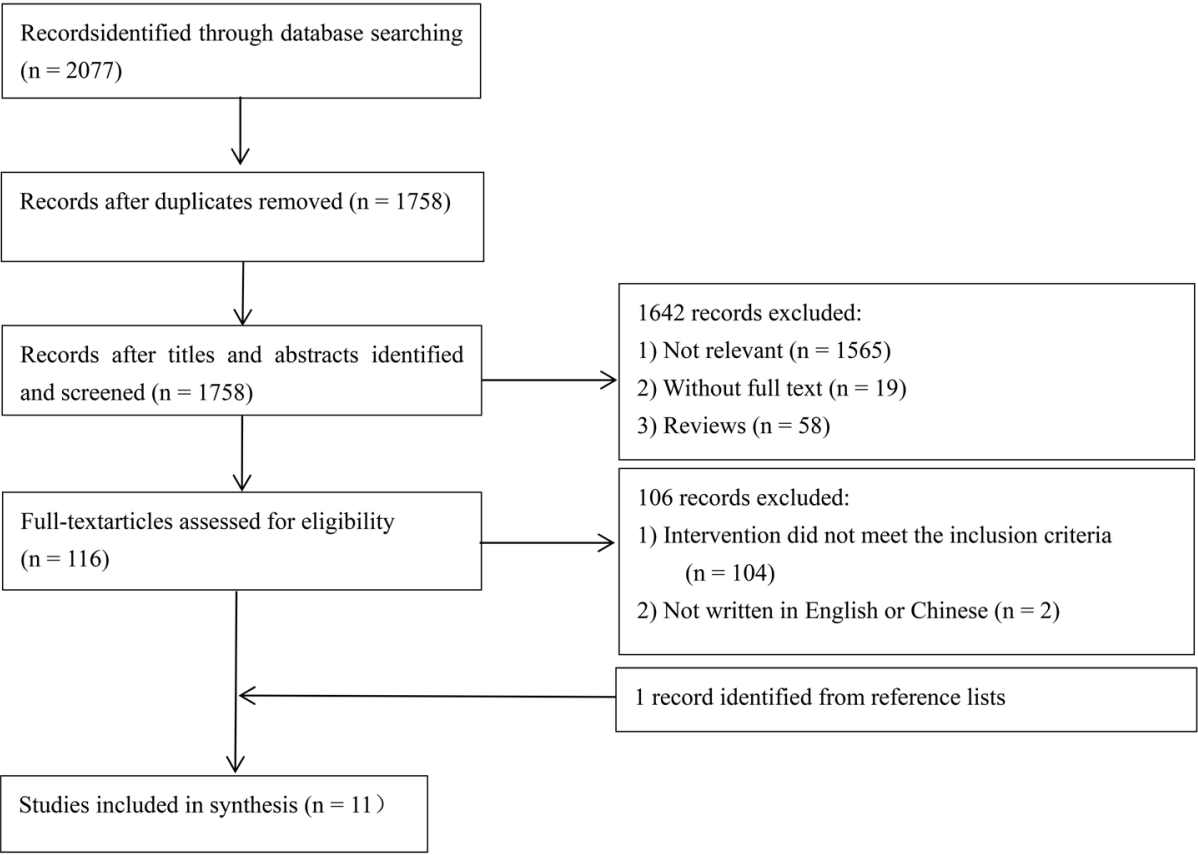

Figure 1. Flow diagram of study selection. 
Table 1. Data extraction of reviewed studies $(\mathrm{N}=11)$.

\begin{tabular}{|c|c|c|c|c|c|c|c|c|}
\hline $\begin{array}{l}\text { Author } \\
\text { Country }\end{array}$ & $\begin{array}{l}\text { Design } \\
\text { setting }\end{array}$ & Participants & $\begin{array}{l}\text { Age } \\
\text { (years) }\end{array}$ & Task & $\begin{array}{l}\text { Course } \\
\text { instructor }\end{array}$ & $\begin{array}{l}\text { Assessment } \\
\text { points }\end{array}$ & $\begin{array}{l}\text { Outcomes and } \\
\text { instruments }\end{array}$ & Results \\
\hline $\begin{array}{l}\text { Hammer } \\
\text { et al. [12] } \\
\text { Denmark }\end{array}$ & $\begin{array}{l}\text { Qualitative study } \\
\text { Setting: } \\
\text { gynecologic } \\
\text { department of } \\
\text { hospital }\end{array}$ & $\begin{array}{l}\text { Patients with } \\
\text { gynecologic } \\
\text { cancer } \\
\mathrm{N}=15\end{array}$ & $\begin{array}{l}\text { Range: } \\
24 \text { - } 87 \\
\text { Median: } \\
\mathrm{m}=52\end{array}$ & $\begin{array}{l}\text { One session } \\
\text { after } \\
\text { interviewing }\end{array}$ & $\begin{array}{l}\text { Not } \\
\text { mentioned }\end{array}$ & & Hope & $\begin{array}{l}\text { All participants } \\
\text { experienced } \\
\text { hope }\end{array}$ \\
\hline $\begin{array}{l}\text { Bar-Sela } \\
\text { et al. [13] } \\
\text { Israel }\end{array}$ & $\begin{array}{l}\text { A non-randomized } \\
\text { controlled trial } \\
\text { Setting: oncology } \\
\text { department of the } \\
\text { hospital }\end{array}$ & $\begin{array}{l}\text { Cancer patients } \\
\text { on chemotherapy } \\
\mathrm{N}=60 \mathrm{~N} 1=19 \\
\text { (intervention } \\
\text { group, patients } \\
\text { who participated } \\
\text { with equal or } \\
\text { more than } 4 \\
\text { sessions) } \\
\mathrm{N} 2=41 \text { (control } \\
\text { group, patients } \\
\text { who participated } \\
\text { with equal or less } \\
\text { than } 2 \text { sessions) }\end{array}$ & $\begin{array}{l}\text { Range: } \\
25-72 \\
\text { Median: } \\
\mathrm{m} 1=55 \\
\mathrm{~m} 2=55\end{array}$ & $\begin{array}{l}\text { An weekly } \\
\text { painting } \\
\text { therapy, } \\
\text { without } \\
\text { mention of } \\
\text { total sessions }\end{array}$ & $\begin{array}{l}\text { More than one } \\
\text { art } \\
\text { therapist }\end{array}$ & $\begin{array}{l}\text { before } \\
\text { each session }\end{array}$ & $\begin{array}{l}\text { 1) HADS } \\
\text { measuring } \\
\text { depression } \\
\text { and anxiety } \\
\text { 2) BFI } \\
\text { measuring } \\
\text { fatigue }\end{array}$ & $\begin{array}{l}\text { Improvements } \\
\text { of depression } \\
(\mathrm{P}=0.021) \text { and } \\
\text { fatigue }(\mathrm{P}=0.24) \\
\text { in the } \\
\text { intervention } \\
\text { group }\end{array}$ \\
\hline $\begin{array}{l}\text { Bozcuk } \\
\text { et al. [14] } \\
\text { Turkey }\end{array}$ & $\begin{array}{l}\text { A non-randomized } \\
\text { controlled trial } \\
\text { Setting: outpatient } \\
\text { chemotherapy unit } \\
\text { and home }\end{array}$ & $\begin{array}{l}\text { Cancer patients } \\
\text { undergoing } \\
\text { chemotherapy } \\
\mathrm{N}=72 \\
\mathrm{~N} 1=48 \\
\text { (intervention } \\
\text { group) } \\
\mathrm{N} 2=24 \\
\text { (control group) }\end{array}$ & $\begin{array}{l}\text { Range: } \\
22-73 \\
\text { Mean: } \\
M=50.6\end{array}$ & $\begin{array}{l}\text { A } 12 \text {-week } \\
\text { course. The } \\
\text { first } 6 \text { weeks } \\
\text { were to get } \\
\text { known the } \\
\text { details of this } \\
\text { study, and the } \\
\text { next } 6 \text { weeks } \\
\text { were offered } \\
\text { painting } \\
\text { therapy }\end{array}$ & $\begin{array}{l}\text { An } \\
\text { experienced } \\
\text { art therapist }\end{array}$ & $\begin{array}{l}\mathrm{t} 1 \text { : before } \\
\text { intervention } \\
\mathrm{t} 2 \text { : after } \\
\text { completing the } \\
\text { intervention }\end{array}$ & $\begin{array}{l}\text { 1) EORTC } \\
\text { QLQ-C30 } \\
\text { measuring } \\
\text { QOL } \\
\text { 2) HADS } \\
\text { measuring } \\
\text { anxiety and } \\
\text { depression }\end{array}$ & $\begin{array}{l}\text { Significant } \\
\text { improvements of } \\
\text { QOL }(\mathrm{P}=0.001) \\
\text { and depression } \\
(\mathrm{P}=0.001) \text { in the } \\
\text { intervention } \\
\text { group }\end{array}$ \\
\hline $\begin{array}{l}\text { Geue et al. } \\
{[15]} \\
\text { Germany }\end{array}$ & $\begin{array}{l}\text { A non-randomized } \\
\text { controlled trial } \\
\text { Setting: } \\
\text { psychosocial } \\
\text { counseling center } \\
\text { in hospital }\end{array}$ & $\begin{array}{l}\text { Patients } \\
\text { with } \\
\text { haematological } \\
\text { malignancies } \\
\mathrm{N}=183 \\
\mathrm{~N} 1=54 \\
\text { (intervention } \\
\text { group) } \\
\mathrm{N} 2=129 \\
\text { (control group) }\end{array}$ & $\begin{array}{l}\text { Range: } \\
\geq 18 \\
\text { Mean: } \\
\text { M1 = } 51.50 \\
\text { M2 }=53.45\end{array}$ & $\begin{array}{l}22 \text { weekly } \\
90 \text {-min } \\
\text { sessions }\end{array}$ & $\begin{array}{l}\text { An artist } \\
\text { receiving } \\
\text { psycho- } \\
\text { oncological } \\
\text { training and } \\
\text { evaluation }\end{array}$ & $\begin{array}{l}\mathrm{t} 1: \text { before } \\
\text { intervention } \\
\mathrm{t} 2 \text { : upon } \\
\text { completing } \\
\text { intervention } \\
\mathrm{t} 3: 6 \text { months } \\
\text { after } \mathrm{t} 2\end{array}$ & $\begin{array}{l}\text { 1) FQCI } \\
\text { measuring } \\
\text { coping with } \\
\text { illness } \\
\text { 2) PACIS } \\
\text { measuring } \\
\text { coping with } \\
\text { illness } \\
\text { 3) HADS } \\
\text { measuring } \\
\text { distress }\end{array}$ & $\begin{array}{l}\text { No effect on } \\
\text { participants' } \\
\text { psychological } \\
\text { distress and } \\
\text { coping behavior }\end{array}$ \\
\hline $\begin{array}{l}\text { Geue et al. } \\
{[16]} \\
\text { Germany }\end{array}$ & $\begin{array}{l}\text { A non-randomized } \\
\text { controlled trial } \\
\text { Setting: } \\
\text { psychosocial } \\
\text { counseling center } \\
\text { in hospital }\end{array}$ & $\begin{array}{l}\text { Cancer patients } \\
\mathrm{N}=53 \\
\mathrm{~N} 1=22 \text { (less } \\
\text { distressed) } \\
\mathrm{N} 2=31 \text { (highly } \\
\text { distressed) }\end{array}$ & $\begin{array}{l}\text { Range: } \\
\geq 18 \\
\text { Mean: } \\
M 1=50 \\
M 2=53\end{array}$ & $\begin{array}{l}22 \text { weekly } \\
90 \text {-min } \\
\text { sessions }\end{array}$ & $\begin{array}{l}\text { An artist } \\
\text { receiving } \\
\text { psycho- } \\
\text { oncological } \\
\text { training }\end{array}$ & $\begin{array}{l}\mathrm{t} 1 \text { : before } \\
\text { intervention } \\
\mathrm{t} 2 \text { : after } \\
\text { completing } \\
\text { the } \\
\text { intervention } \\
\mathrm{t} 3: 6 \text {-months } \\
\text { after } \mathrm{t} 2\end{array}$ & $\begin{array}{l}\text { EORTC } \\
\text { QLQ-C30 } \\
\text { measuring } \\
\text { QOL }\end{array}$ & $\begin{array}{l}\text { No effect on } \\
\text { QOL on } \\
\text { participants but } \\
\text { improvements } \\
\text { of role, } \\
\text { emotional and } \\
\text { social } \\
\text { functioning and } \\
\text { fatigue in the } \\
\text { group of highly } \\
\text { distressed } \\
\text { patients }\end{array}$ \\
\hline
\end{tabular}




\section{Continued}

\begin{tabular}{|c|c|c|c|c|c|c|c|c|}
\hline $\begin{array}{l}\text { Oster et al. } \\
{[17]} \\
\text { Sweden }\end{array}$ & $\begin{array}{l}\text { A randomized } \\
\text { controlled trial } \\
\text { Setting: oncology } \\
\text { department of the } \\
\text { hospital }\end{array}$ & $\begin{array}{l}\text { Women with } \\
\text { non-metastatic } \\
\text { breast cancer } \\
\mathrm{N}=41 \mathrm{~N} 1=20 \\
\text { (intervention } \\
\text { group) } \\
\mathrm{N} 2=21 \\
\text { (control group) }\end{array}$ & $\begin{array}{l}\text { Range: } \\
37-69 \\
\text { Median: } \\
\mathrm{m} 1=59.5 \\
\mathrm{~m} 2=55\end{array}$ & $\begin{array}{l}5 \text { weekly } \\
\text { sessions after } \\
\text { postoperative } \\
\text { radiotherapy }\end{array}$ & $\begin{array}{l}\text { Two trained } \\
\text { art therapists }\end{array}$ & $\begin{array}{l}\mathrm{t} 1 \text { : before the } \\
\text { start of } \\
\text { radiotherapy } \\
\mathrm{t} 2: 2 \text { months } \\
\text { after } \\
\text { radiotherapy } \\
\text { t3: } 6 \text { months after } \\
\text { radiotherapy }\end{array}$ & $\begin{array}{l}\text { CRI } \\
\text { measuring } \\
\text { coping } \\
\text { resources }\end{array}$ & $\begin{array}{l}\text { An overall } \\
\text { increase of } \\
\text { coping resources } \\
\text { in the } \\
\text { intervention } \\
\text { group }\end{array}$ \\
\hline $\begin{array}{l}\text { Singer et } \\
\text { al. [18] } \\
\text { Germany }\end{array}$ & $\begin{array}{l}\text { A non-randomized } \\
\text { controlled trial } \\
\text { Setting: an } \\
\text { outpatient } \\
\text { psycho-oncology } \\
\text { unit }\end{array}$ & $\begin{array}{l}\text { Patients } \\
\text { with } \\
\text { haematological } \\
\text { malignancies } \\
\mathrm{N}=165 \mathrm{~N} 1=36 \\
\text { (intervention } \\
\text { group) } \mathrm{N} 2=129 \\
\text { (control group) }\end{array}$ & $\begin{array}{l}\text { Range: } \\
\geq 18\end{array}$ & $\begin{array}{l}22 \text { weekly } \\
90 \text {-min } \\
\text { sessions }\end{array}$ & $\begin{array}{l}\text { An artist } \\
\text { receiving } \\
\text { psychological } \\
\text { training and } \\
\text { evaluation }\end{array}$ & $\begin{array}{l}\mathrm{t} 1 \text { : before } \\
\text { intervention } \\
\mathrm{t} 2 \text { : immediately } \\
\text { after } \\
\text { intervention }\end{array}$ & $\begin{array}{l}\text { SRGS } \\
\text { measuring } \\
\text { post-traumatic } \\
\text { growth }\end{array}$ & $\begin{array}{l}\text { No difference in } \\
\text { post-traumatic } \\
\text { growth between } \\
\text { groups }\end{array}$ \\
\hline $\begin{array}{l}\text { Peng et al. } \\
{[19]} \\
\text { China }\end{array}$ & $\begin{array}{l}\text { A non-randomized } \\
\text { controlled trial } \\
\text { Setting: inpatient } \\
\text { ward of the } \\
\text { hospital }\end{array}$ & $\begin{array}{l}\text { Patients with } \\
\text { breast cancer } \\
\text { undergoing } \\
\text { chemotherapy } \\
\mathrm{N}=200\end{array}$ & $\begin{array}{l}\text { Range: } \\
\geq 18 \\
\text { Mean: } \\
\mathrm{M}=45.35\end{array}$ & $\begin{array}{l}\text { Painting } \\
\text { during the } \\
\text { day of the } \\
\text { second } \\
\text { chemotherapy } \\
\text { lasted for } \\
2-4 \mathrm{~h}\end{array}$ & $\begin{array}{l}\text { The } \\
\text { researchers } \\
\text { themselves }\end{array}$ & $\begin{array}{l}\mathrm{t} 1: 24 \mathrm{~h} \text { after first } \\
\text { chemotherapy } \\
\mathrm{t} 2: 24 \mathrm{~h} \text { after } \\
\text { painting therapy }\end{array}$ & $\begin{array}{l}\text { 1) FLIE } \\
\text { measuring life } \\
\text { function } \\
\text { 2) SAS } \\
\text { measuring } \\
\text { anxiety }\end{array}$ & $\begin{array}{l}\text { Significant } \\
\text { improvements of } \\
\text { life function } \\
(\mathrm{P}<0.001) \\
\text { and anxiety } \\
(\mathrm{P}<0.001) \text { in } \\
\text { the intervention } \\
\text { group }\end{array}$ \\
\hline $\begin{array}{l}\text { Svensk } \\
\text { et al. } \\
{[20]} \\
\text { Sweden }\end{array}$ & $\begin{array}{l}\text { A randomized } \\
\text { controlled trial } \\
\text { Setting: oncology } \\
\text { department of the } \\
\text { hospital }\end{array}$ & $\begin{array}{l}\text { Women with } \\
\text { non-metastatic } \\
\text { breast cancer } \\
\mathrm{N}=41 \mathrm{~N} 1=20 \\
\text { (intervention } \\
\text { group) } \\
\mathrm{N} 2=21 \\
\text { (control group) }\end{array}$ & $\begin{array}{l}\text { Range: } \\
37-69 \\
\text { Median: } \\
\mathrm{m} 1=59.5 \\
\mathrm{~m} 2=55\end{array}$ & $\begin{array}{l}5 \text { weekly } \\
\text { sessions after } \\
\text { postoperative } \\
\text { radiotherapy }\end{array}$ & $\begin{array}{l}\text { Two trained } \\
\text { art therapists }\end{array}$ & $\begin{array}{l}\mathrm{t} 1 \text { : before the } \\
\text { start of } \\
\text { radiotherapy } \\
\mathrm{t} 2: 2 \text { months } \\
\text { after } \\
\text { radiotherapy } \\
\text { t3: } 6 \text { months } \\
\text { after } \\
\text { radiotherapy }\end{array}$ & $\begin{array}{l}\text { 1) } \\
\text { WHOQOL-BR } \\
\text { EF measuring } \\
\text { QOL } \\
\text { 2) QLQ-BR23 } \\
\text { measuring } \\
\text { QOL }\end{array}$ & $\begin{array}{l}\text { An overall } \\
\text { improvement of } \\
\text { QOL in the } \\
\text { intervention } \\
\text { group }\end{array}$ \\
\hline $\begin{array}{l}\text { Thyme } \\
\text { et al. } \\
{[21]} \\
\text { Sweden }\end{array}$ & $\begin{array}{l}\text { A randomized } \\
\text { controlled trial } \\
\text { Setting: oncology } \\
\text { department of the } \\
\text { hospital }\end{array}$ & $\begin{array}{l}\text { Women with } \\
\text { nonmetastatic } \\
\text { breast cancer } \\
\mathrm{N}=42 \\
\mathrm{~N} 1=20 \\
\text { (intervention } \\
\text { group) } \\
\mathrm{N} 2=22 \\
\text { (control group) }\end{array}$ & $\begin{array}{l}\text { Range: } \\
37-69 \\
\text { Median: } \\
\mathrm{m} 1=59.50 \\
\mathrm{~m} 2=55\end{array}$ & $\begin{array}{l}5 \text { weekly } \\
\text { sessions given } \\
\text { after } \\
\text { postoperative } \\
\text { radiotherapy }\end{array}$ & $\begin{array}{l}\text { Two trained } \\
\text { art therapists }\end{array}$ & $\begin{array}{l}\mathrm{t} 1 \text { : before } \\
\text { intervention, } \\
\mathrm{t} 2 \text { : following } \\
\text { intervention } \\
\text { t3: a 4-month } \\
\text { follow-up }\end{array}$ & $\begin{array}{l}\text { 1) SASB } \\
\text { measuring } \\
\text { self-image } \\
\text { 2) SCL-90 } \\
\text { measuring } \\
\text { psychiatric } \\
\text { symptoms }\end{array}$ & $\begin{array}{l}\text { Significant } \\
\text { improvements } \\
\text { of psychiatric } \\
\text { symptoms } \\
(\mathrm{P}<0.05) \text { but } \\
\text { no significant } \\
\text { change on } \\
\text { self-image in the } \\
\text { intervention } \\
\text { group }\end{array}$ \\
\hline $\begin{array}{l}\text { Zhao \& } \\
\text { Tang [22] } \\
\text { China }\end{array}$ & $\begin{array}{l}\text { A randomized } \\
\text { controlled trial } \\
\text { Setting: } \\
\text { demonstration } \\
\text { classroom of the } \\
\text { inpatient ward }\end{array}$ & $\begin{array}{l}\text { Cancer patients } \\
\mathrm{N}=86 \\
\mathrm{~N} 1=43 \\
\text { (mandala } \\
\text { drawing therapy) } \\
\mathrm{N} 2=43 \text { (routine } \\
\text { nursing care) }\end{array}$ & $\begin{array}{l}\text { Mean: } \\
\text { M1 = 53.3 } \\
\text { M2 = 54.3 }\end{array}$ & $\begin{array}{l}2 \text { times per } \\
\text { week for } 6 \\
\text { weeks, } 45 \\
\text { minutes each } \\
\text { time }\end{array}$ & $\begin{array}{l}\text { Members } \\
\text { receiving } \\
\text { training and } \\
\text { evaluation of } \\
\text { mandala } \\
\text { painting } \\
\text { psychotherapy }\end{array}$ & $\begin{array}{l}\mathrm{t} 1: \text { before } \\
\text { intervention } \\
\mathrm{t} 2: 2 \text { weeks after } \\
\text { intervention } \\
\mathrm{t} 3: 4 \text { weeks after } \\
\text { intervention } \\
\text { t4: } 6 \text { weeks after } \\
\text { intervention }\end{array}$ & $\begin{array}{l}\text { SAS } \\
\text { measuring } \\
\text { anxiety }\end{array}$ & $\begin{array}{l}\text { Significant } \\
\text { improvement of } \\
\text { anxiety }(\mathrm{P}<0.05) \\
\text { in the } \\
\text { intervention } \\
\text { group }\end{array}$ \\
\hline
\end{tabular}

Abbreviation: EORTC QLQ-C30: European Organization for the Research and Treatment of Cancer Quality of Life Core Questionnaire; HADS: Hospital Anxiety and Depression Scale. FLIE: Functional Living Index-Emesis; SAS: Self-Rating Anxiety Scale; SRGS: Stress-Related Growth Scale; FQCI: Freiburg Questionnaire on Coping with Illness; PACIS: Perceived Adjustment to Chronic Illness Scale; SASB: The Structural Analysis of Social Behavior; SCL-90: The Symptom Check List-90; WHOQOL-BREF: The World Health Organization Quality of Life Instruments; EORTC QLQ-BR23: The European Organization for Research and Treatment of Cancer (EORTC) Breast-Cancer-Specific Quality of Life Questionnaire; BFI: The Brief Fatigue Inventory; CRI: The Coping Resources Inventory; QOL: Quality of Life; N: The Number of Participants; M: Mean Age; m: Median Age; t: Assessment Points. 
The types of cancer included breast cancer, hematological tumor, multiple myeloma, Non-Hodgkin lymphoma, acute leukaemia, chronic leukaemia, Hodgkin lymphoma, gynecologic cancer, esophageal cancer and lung cancer. A total of 913 participants consisted of 630 women and 283 men at different stage of cancer were included.

In terms of study quality, the scores for quality evaluation ranged from 230 350 (see Table 2). Regretfully, the quality assessment tool only provided scoring without giving criterion to classify studies into "high quality" "median quality" or "low quality". The most common quality limitation in the included studies were: ethical issues and bias addressed too brief or overlooked, and descriptions of intervention were incomplete.

\subsubsection{Setting}

All included studies reported setting. These studies occurred in psycho-social counselling center $(\mathrm{N}=2)$, outpatient unit $(\mathrm{N}=2)$ and inpatient unit $(\mathrm{N}=7)$. Besidesasking patients to draw during chemotherapy administration, the study of Bozcuk also provided patients painting materials for home study at their convenience [14].

\subsubsection{Therapy Sessions}

There was considerable heterogeneity regarding content, format of the painting therapy interventions. Frequency of intervention varied from 1 single session to 22 weekly sessions. Length of each session varied from 45 minutes to 4 hours, however, 7 studies just reported the number of sessions without reporting course duration (see Table 1). Most painting therapies are composed of several sessions although there is no universally applicable standard procedure. Usually, the sessions took place in groups of mixed gender and age and consisted of 5 to 8 patients each group [15] [16] [18].

Table 2 . Research quality appraisal $(\mathrm{N}=11)$.

\begin{tabular}{|c|c|c|c|c|c|c|c|c|c|c|}
\hline Author & $\begin{array}{l}\text { Abstract } \\
\text { and title }\end{array}$ & $\begin{array}{l}\text { Introduction } \\
\text { and aims }\end{array}$ & $\begin{array}{l}\text { Method } \\
\text { and data }\end{array}$ & Sampling & $\begin{array}{c}\text { Data } \\
\text { analysis }\end{array}$ & $\begin{array}{l}\text { Ethics } \\
\text { and bias }\end{array}$ & Results & $\begin{array}{l}\text { Transferability } \\
\text { or } \\
\text { generalizability }\end{array}$ & $\begin{array}{c}\text { Implications } \\
\text { and } \\
\text { usefulness }\end{array}$ & Total \\
\hline Hammer et al. [12] & 40 & 40 & 30 & 40 & 40 & 40 & 40 & 40 & 40 & 350 \\
\hline Bar-Sela et al. [13] & 40 & 40 & 30 & 40 & 40 & 40 & 40 & 40 & 40 & 350 \\
\hline Bozcuk et al. [14] & 40 & 30 & 30 & 30 & 40 & 10 & 40 & 30 & 40 & 290 \\
\hline Geue et al. [15] & 30 & 40 & 40 & 40 & 30 & 40 & 40 & 40 & 30 & 330 \\
\hline Geue et al. [16] & 40 & 40 & 40 & 30 & 40 & 20 & 40 & 40 & 40 & 330 \\
\hline Oster et al. [17] & 30 & 40 & 30 & 40 & 40 & 30 & 40 & 40 & 40 & 330 \\
\hline Singer et al. [18] & 40 & 40 & 40 & 40 & 40 & 40 & 40 & 40 & 30 & 350 \\
\hline Peng et al. [19] & 40 & 20 & 40 & 30 & 40 & 30 & 40 & 40 & 30 & 310 \\
\hline Svensk et al. [20] & 40 & 30 & 30 & 40 & 30 & 40 & 40 & 40 & 40 & 330 \\
\hline Thyme et al. [21] & 40 & 30 & 30 & 40 & 30 & 40 & 40 & 40 & 40 & 330 \\
\hline Zhao \& Tang [22] & 30 & 30 & 30 & 30 & 20 & 10 & 30 & 30 & 20 & 230 \\
\hline
\end{tabular}


The included studies reported phased tasks with aims and methods during painting therapy. In Geue's study [16], the intervention was consisted of 3 phases: participants learned use of material and practiced experimental drawing in phase I (session 1 - 7); then participants started to express their ideas and make use of watercolor in phase II (session 8 - 13); finally, participants created a book telling their own stories in phase III (session 14 - 21). Painting therapy in Bozcuk's study [14] lasted for 12 weeks. The first 6 weeks sessions were provided to discern study details. After that, participants were offered to take part in the study. In Singer's study [18], at the beginning of the sessions, participants were requested to perform simple drawing techniques to make them feel comfortable with the situation. Started from the sixth session, participants gradually intended to explore and express their feelings by drawing their current mood or by painting a self-portrait. In the final phase of the program, patients were encouraged to create their ideas about the disease and their coping processes. In Thyme's study [21], the first session of painting therapy focused on visualization of feelings through analogue drawings. The women were encouraged to choose one or several of her analogue drawings and make one or several images. The second session focused on life-size body outline. Participants painted different feelings in colors, figures, and forms within the outline in the different parts of body. During the third and fourth sessions, the participant painted whatever they wanted in several pictures. Final session was to make a conclusion. At the first meeting of Bar-Sela's intervention [13], patients were asked to paint at least three paintings spontaneously, without any instructions at the first meeting. The art therapist tried to make a diagnosis of patient's mental situation through three paintings. Started from the second meeting, the therapist started to give the patients guidance including the use of color, subjects of drawing and technical options.

A participant in painting therapy need not know any knowledge of painting. Participants' works were finished in three ways: painting freely, painting according to specific theme and coloring picture. In four studies [14] [16] [18] [20], the participants decided on what they wanted to paint and how to express their inner pictures. In five studies [12] [13] [15] [17] [21], participants painted according to the given themes. Participants in two studies were asked to color picture [19] [22]. In Peng's study [19], painting album “Secret Garden” of British illustrator Johanna Basford was provided to color. In order to increase the interests of patients and avoid the difficulty with drawing, patients feel free to choose their own favorite pictures to color. Zhao [22] provided pencils for patients to color the given mandala graphics. Painting materials provided includes color pencil, watercolors, lead pencils, oil pastel, charcoals, tape, paintbrush, sheets of paper and paper rolls.

Most of the included studies have involved one or more art therapists except studies of Peng [19], Zhao [22] and Hammer [12]. In Peng's study [19], the researchers guided the sessions. In Zhao's study [22], members from psychological 
nursing group receiving painting psychotherapy training and being qualified guided the sessions. Hammer's qualitative study [12] did not report any art therapist.

\subsubsection{Effects of Painting Therapy}

Quality of life, coping with disease (post-traumatic growth, coping with illness, self-image), mental health (anxiety, depression and hope) and somatic symptoms (life function, fatigue) were reported in the included studies. Positive effects of painting therapy on health outcomes were reported in 9 studies [12] [13] [14] [16] [17] [19] [20] [21] [22]. Negative effects of painting therapy on quality of life, coping with disease and mental health were reported in 4 studies [15] [16] [18] [21].

Three studies reported quality of life as an outcome (Table 1). Two of them reported significant improvements of the quality of life in the intervention group with painting therapy [14] [20]. The remaining one presented no significant difference in quality of life between patients either with high or low levels of distress received painting therapy [16], however, improvements in the group of highly distressed patients in terms of role, emotional and social functioning and fatigue were discovered.

Four studies reported coping with illness as an outcome (Table 1), of which only one reported an overall increase of coping resources among patients at 2 months and 6 months after baseline measurement [17]. The remaining 3 studies [15] [18] [21] presented no significant improvement in post-traumatic growth, coping with illness or self-image. However, participants in the study of Geue [16] expressed positive subjective experiences through the whole intervention process.

Six studies reported mental health as an outcome (Table 1). Among these studies, 4 reported significant improvements of depression and anxiety [14] [19] [21] [22]. The qualitative study [12] concentrating on how patients express the meanings of hope in drawing highlighted that painting therapy gave a new understanding of the experience of hope. The rest one had no detectable effect on participants' psychological anxiety and depression [15].

Two studies reported somatic symptom as an outcome (Table 1 ) showing significant improvement in life function $(\mathrm{P}<0.001)[19]$ and improvement in fatigue to a lesser degree [13].

\section{Discussion}

Strong evidence of positive effects of painting therapy on cancer patients' quality of life, coping with disease, mental health and somatic symptom were detected. Although some inconsistencies still exist, there is no evidence that painting therapy causes harm.

The reasons why painting therapy influences cancer patients' health have been presented in the included studies in this review. Here are some tentative interpretations. Firstly, painting can serve as a tool to express the emotion, thoughts, 
feelings and conflict, especially for those who do not have the chance to express their thoughts in words [23]. Secondly, as an initial communication mean of human, painting is less defensive which uses symbolic language to bypass people's potential psychological defense mechanism, and involuntarily projects inner feelings, anxiety, contradictions, values, desires into the works, so that hidden or suppressed emotions can be released [24]. Thirdly, painting therapy combining actions and reflections played a necessary role to help promote participants process of understanding and current situation [25]. Drawing could bring mixed, poorly understood feelings to order and clarity [26] [27]. One brings together diverse elements of their experience to make a new and meaningful whole in the process of drawing [28]. In addition, being in a group, where social interaction is possible and valued, social support and membership can help with the patients' quality of life [14] [18].

Possible explanations for inconsistency could be small sample size, heterogeneity in cancer population, measuring instrument and statistical methods [15] [16] [18]. Randomized trials in this topic are few and only 4 randomized controlled trials were retrieved in this review. Non-randomization leads to differences, concerning baseline data in experimental and control groups. Even if known confounders can be controlled, residual confounding could still exist. What's more, there is a lack of theoretical framework to guide research. Therefore, more randomized trials with theoretical framework are needed in future studies. Besides, the number of participants enrolled in the included studies was commonly small, larger populations are necessary to conform the results of painting therapy. The use of different questionnaires could be potential factor of inconsistent findings. As a result, the measuring instruments used in future should be sensitive enough to measure changes.

Art therapist, playing an important role in the intervention, gives patients guidance including use of colors, subjects of drawing and technical options [20], and never functions as both interviewer and therapist for the same patient. When there was an interview, to make the participants feel free to express both negative and positive experiences about the participation, the interview should be conducted by another art therapist who did not leading therapy sessions [13]. The function of art therapist is to motivate each participant to attend the sessions regularly, and facilitate talks within a group about the completed paintings and express their feelings and thoughts [18] [20]. Future study on painting therapy should have professional art therapist involved in.

As a form of art therapy, painting therapy is suitable for patients with various diagnoses, ages and levels of education [29] [30]. Places for painting therapy intervention are flexible to choose. This review included cancer population in various cancer stage and more women than man participated in painting therapy was discovered. An explanation that male cancer patients often try to cope with disease in an internal way or only with their life partners may give evidence to this phenomenon [31]. Future study should pay more attention to explore this 
topic on male cancer population. Since coping with cancer is a process beyond the completion of medical treatments, future studies should examine at which time (acute, rehabilitative and outpatient care) painting therapy is most useful.

Although Geue et al. [15] failed to find benefit to participants in the painting therapy intervention, however, participants described their experience were positive throughout. Since quantitative data cannot give insight into processes underlying assessments in questionnaires and it is difficult to express subjective concept of quality of life in a quantitative and objective way [32], qualitative data should be considered to be included in the future to give more evidence on the exploration of painting therapy.

Geue et al. [16] and Bozcuk et al. [14] discovered that patients with poorer well-being were the ones that had better improvements by participating in painting therapy. This finding suggests that baseline levels of well-being should be taken into account when implementing a painting therapy. Painting therapy may be expected to benefit those who are relatively in more need of help in clinical practice. It is sensible to prioritize cancer patients who have the greatest need when financial and time resources are limited.

Considering that some participants found it hard to work in a group [13], creating individual session with patients in addition to group session is a practical suggestion. As there was considerable heterogeneity in the design of painting therapy interventions, different modes should be compared and perfect in future studies.

\subsection{Limitations}

There were some limitations in this review. First, narrative synthesis was conducted owing to heterogeneity, which limited generalizability. Second, because of the small amount of literature on this topic, 3 studies extending from a same sample were all included which may cause the research outcomes less persuasive. Last, no effect size was presented because both quantitative and qualitative studies were included.

\subsection{Implications}

The study is the latest and the most comprehensive systematic review on paining therapy dependently in cancer population up to now. Various forms of painting therapy in cancer population have been summed up. The effects on participants have been analyzed. As an economical and convenient method, painting therapy is feasible in various types of cancer populations with positive effects on participants quality of life, coping with disease, mental health and somatic symptoms. We call for more randomized controlled trials with larger sample size in future studies. The effects on participants at different cancer stage and who benefits most should be explored. Studies on male participants should be paid attention to. Besides, different modes of painting therapy should be developed and compared. 


\section{Conclusion}

This systematic review synthesizes the latest research evidence of painting therapy in adult cancer patients, lying a groundwork for future research in clinics. The results from the current study strongly support painting therapy as a powerful tool in rehabilitation of various types of cancer patients and painting therapy is feasible in daily practice, although the examination of the effectiveness of painting therapy in psycho-oncology is still relative young.

\section{Conflicts of Interest}

The authors declare no conflicts of interest regarding the publication of this paper.

\section{References}

[1] Dalley, T. (2008) Art as Therapy: An Introduction to the Use of Art as a Therapeutic Technique. Routledge, Abingdon.

[2] Rubin, J.A. (1999) Art Therapy: An Introduction. Psychology Press.

[3] Visser, A. and Op'T Hoog, M. (2008) Education of Creative Art Therapy to Cancer Patients: Evaluation and Effects. Journal of Cancer Education, 23, 80-84.

https://doi.org/10.1080/08858190701821204

[4] Vick, R.M. (2003) A Brief History of Art Therapy. In: Handbook of Art Therapy, 5-15.

[5] Malchiodi, C.A. (2012) Art Therapy and Health Care. Guilford Press, New York.

[6] Gough, W.C. (1986) Arts and Flowers: Drawing out the Patient's Best. A Growing Interest. The American Journal of Nursing, 86, 164.

[7] Geue, K., Goetze, H., Buttstaedt, M., Kleinert, E., Richter, D. and Singer, S. (2010) An Overview of Art Therapy Interventions for Cancer Patients and the Results of Research. Complementary Therapies in Medicine, 18, 160-170. https://doi.org/10.1016/j.ctim.2010.04.001

[8] Liberati, A., Altman, D.G., Tetzlaff, J., Mulrow, C., Gotzsche, P.C., Ioannidis, J.P., Moher, D., et al. (2009) The PRISMA Statement for Reporting Systematic Reviews and Meta-Analyses of Studies That Evaluate Health Care Interventions: Explanation and Elaboration. Journal of Clinical Epidemiology, 62, e1-e34. https://doi.org/10.1016/j.jclinepi.2009.06.006

[9] Hawker, S., Payne, S., Kerr, C., Hardey, M. and Powell, J. (2002) Appraising the Evidence: Reviewing Disparate Data Systematically. Qualitative Health Research, 12, 1284-1299. https://doi.org/10.1177/1049732302238251

[10] Eysenck, H.J. (1995) Problems with Meta-Analysis. In: Chalmers, I. and Altman, D.G., Eds., Systematic Reviews, BMJ Publishing, London, 64-74.

[11] Petticrew, M. and Roberts, H. (2006) Systematic Reviews in the Social Sciences: A Practical Guide.

[12] Hammer, K., Hall, E.O.C. and Mogensen, O. (2013) Hope Pictured in Drawings by Women Newly Diagnosed with Gynecologic. Cancer Nursing, 36, E42-E50. https://doi.org/10.1097/NCC.0b013e31826c7af2

[13] Bar-Sela, G., Atid, L., Danos, S., Gabay, N. and Epelbaum, R. (2007) Art Therapy Improved Depression and Influenced Fatigue Levels in Cancer Patients on Chemotherapy. Psycho-Oncology, 16, 980-984. https://doi.org/10.1002/pon.1175 
[14] Bozcuk, H., Ozcan, K., Erdogan, C., Mutlu, H., Demir, M. and Coskun, S. (2017) A Comparative Study of Art Therapy in Cancer Patients Receiving Chemotherapy and Improvement in Quality of Life by Watercolor Painting. Complementary Therapies in Medicine, 30, 67-72. https://doi.org/10.1016/j.ctim.2016.11.006

[15] Geue, K., Richter, R., Buttstaedt, M., Braehler, E. and Singer, S. (2013) An Art Therapy Intervention for Cancer Patients in the Ambulant Aftercare-Results from a Non-Randomised Controlled Study. European Journal of Cancer Care, 22, 345-352. https://doi.org/10.1111/ecc.12037

[16] Geue, K., Rieckhof, S., Buttstaedt, M. and Singer, S. (2017) Do Cancer Patients with High Levels of Distress Benefit More than Less Distressed Patients from Outpatient Art Therapy? European Journal of Oncology Nursing, 30, 1-7.

[17] Oster, I. (2006) Art Therapy with Women with Breast Cancer-Results from a Randomized Study. Psycho-Oncology, 18, S22-S23.

[18] Singer, S., Gotze, H., Buttstadt, M., Ziegler, C., Richter, R., Brown, A., Geue, K., et al. (2013) A Non-Randomised Trial of an Art Therapy Intervention for Patients with Haematological Malignancies to Support Post-Traumatic Growth. Journal of Health Psychology, 18, 939-949. https://doi.org/10.1177/1359105312458332

[19] Peng, X., Chen, J., Huang, L., Wen, Q., Yan, Q.Y. and Du, S.J. (2017) Study on the Effect of Painting Therapy on Breast Cancer Patients Undergoing Chemotherapy. Chinese Journal of Nursing, 10, 1200-1203.

[20] Svensk, A.C., Oster, I., Thyme, K.E., Magnusson, E., Sjodin, M., Eisemann, M., Lindh, J., et al. (2009) Art Therapy Improves Experienced Quality of Life among Women Undergoing Treatment for Breast Cancer: A Randomized Controlled Study. European Journal of Cancer Care, 18, 69-77. https://doi.org/10.1111/j.1365-2354.2008.00952.x

[21] Thyme, K.E., Sundin, E.C., Wiberg, B., Oster, I., Astrom, S., Lindh, J., et al. (2009) Individual Brief Art Therapy Can Be Helpful for Women with Breast Cancer: A Randomized Controlled Clinical Study. Palliat Support Care, 7, 87-95. https://doi.org/10.1017/S147895150900011X

[22] Zhao, S. and Tang, F. (2017) Effect of Mandala Painting Psychotherapy on Anxiety of Cancer Patients. Tianjin Nursing, 6, 505-506.

[23] Durualp, E. and Altay, N. (2012) A Comparison of Emotional Indicators and Depressive Symptom Levels of School-Age Children with and without Cancer. Journal of Pediatric Oncology Nursing, 29, 232-239. https://doi.org/10.1177/1043454212446616

[24] Katz, C. and Hamama, L. (2013) "Draw Me Everything That Happened to You": Exploring Children's Drawings of Sexual Abuse. Children and Youth Services Review, 35, 877-882. https://doi.org/10.1016/j.childyouth.2013.02.007

[25] Sugarman, A. (2006) Mentalization, Insightfulness, and Therapeutic Action: The Importance of Mental Organization. The International Journal of Psychoanalysis, 87, 965-987. https://doi.org/10.1516/6DGH-0KJT-PA40-REX9

[26] Bentley, T. (1989) Talking Pictures. Nursing Times, 85, 58-59.

[27] Dalley, T., Case, C., Schaverien, J., Weir, F., Halliday, D., Hall, P.N. and Waller, D. (1987) Images of Art Therapy. Tavistock, London.

[28] Khadar, M.G., Babapour, J. and Sabourimoghaddam, H. (2013) The Effect of Art Therapy Based on Painting Therapy in Reducing Symptoms of Oppositional Defiant Disorder (odd) in Elementary School Boys. Procedia-Social and Behavioral Sciences, 84, 1872-1878. https://doi.org/10.1016/j.sbspro.2013.07.051 
[29] Nainis, N.A. (2008) Approaches to Art Therapy for Cancer Inpatients: Research and Practice Considerations. Art Therapy, 25, 115-121. https://doi.org/10.1080/07421656.2008.10129597

[30] Goetze, H., Geue, K., Buttstädt, M., Singer, S. and Schwarz, R. (2009) Art Therapy for Cancer Patients in Outpatient Care. Psychological Distress and Coping of the Participants. Forschende Komplementarmedizin, 16, 28-33.

[31] Okuyama, T., Endo, C., Seto, T., Kato, M., Seki, N., Akechi, T., Hosaka, T., et al. (2008) Cancer Patients' Reluctance to Disclose Their Emotional Distress to Their Physicians: A Study of Japanese Patients with Lung Cancer. Psycho-Oncology. Journal of the Psychological, Social and Behavioral Dimensions of Cancer, 17, 460-465. https://doi.org/10.1002/pon.1255

[32] Shimozuma, K., Okamoto, T., Katsumata, N., Koike, M., Tanaka, K., Osumi, S., Yamauchi, C., et al. (2002) Systematic Overview of Quality of Life Studies for Breast Cancer. Breast Cancer, 9, 196-202. https://doi.org/10.1007/BF02967589 